\title{
COMPETENCIAS DEL DOCENTE UNIVERSITARIO COMO GUÍA EN EL ESPACIO EUROPEO DE EDUCACIÓN SUPERIOR
}

\author{
UNIVERSITY TEACHER'S COMPETENCE AS A GUIDE IN THE EUROPEAN HIGHER \\ EDUCATION
}

\author{
Alfonso Javier García González \\ Yolanda Troyano Rodríguez \\ Universidad de Sevilla \\ Luís Sérgio Vieira \\ Universidade do Algarve
}

Fecha de recepción: 12 de junio de 2013
Fecha de aceptación: 15 de junio de 2014
Fecha de publicación: 30 de junio de 2014

\section{RESUMEN}

El presente trabajo pretende conocer la opinión de alumnado universitario, de diferentes cursos académicos, en relación con las competencias que ha de desarrollar un tutor para ser considerado excelente. El personal docente se encuentra en el transcurso de una adaptación que le ha de permitir interactuar, de manera eficiente, en diferentes situaciones. Una faceta de este entorno es la acción tutorial, representada en la enseñanza universitaria en la figura del profesor tutor y desarrollada en su relación con el alumnado. Se ha empleado el método cualitativo Delphi para analizar las expectativas del alumnado. A lo largo de sucesivas rondas, se han obtenido una serie de competencias con que, según los expertos consultados, debería contar el profesorado que ejercerá la acción tutorial en el contexto del EEES. Como conclusión principal destaca que, además de las competencias demandadas, se observa que éstas varían en función del curso al que pertenece el alumnado participante.

Palabras clave: Orientación universitaria; Acción tutorial; Competencias tutor; Delphi; Excelencia.

\begin{abstract}
This paper aims to ascertain the opinion of university students from different academic years, in relation to the powers of developing a tutor has to be considered excellent. The teacher is in the course of an adaptation that should allow you to interact, efficiently in different situations. One facet of this is the tutorial environment, represented in higher education in the tutor and developed in their relationship with the student teacher. It has used the Delphi method to analyze the qualitative expectations of students. Over successive rounds, they have been obtained with a set of skills that, according to the experts consulted, should have teachers who exercise the tutorial action in the context of the EHEA. The main conclusion emphasizes that, in addition to the skills demanded, it is observed that they vary depending on the course to which the student belongs participant.
\end{abstract}

Key words: College Counselling; Tutorial Action; Skills Tutor; Delphi; Excellence. 
Competencias del docente universitario como guía en el espacio europeo de educación superior

\section{INTRODUCCIÓN}

La tarea docente implica un conjunto de actividades dependientes entre sí, que necesita estrategias de apoyo y coordinación con otros docentes. Del mismo modo, la acción tutorial, como actividad inherente de la tarea docente, desde su planificación, puesta en marcha y posterior evaluación, requiere de una acción perfectamente coordinada para obtener resultados que puedan definirse como de calidad. A tal fin el profesorado tendrá que incluir entre sus competencias docentes las competencias tutoriales. Y es que el personal docente como planteaba Lázaro (1997) no debe ser sólo un "conocedor de la ciencia, un experto en técnicas y un investigador, sino que ha de ser guía y supervisor de la formación científica del estudiante".

Para asumir el reto planteado por Lázaro a finales de los 90 es preciso, a su vez, asumir riesgos. Pues el riesgo profesional es inevitable, porque el proceso de formación se decide en los contextos sociales de interacción. Asimismo, los procesos de formación institucionales se presentan a los educadores, frecuentemente, con la intencionalidad quebrada por las circunstancias vitales y de historia cultural de los actores (García, 2012). Y en dichos procesos formativos entran las funciones del tutor, que han ido evolucionando y adquiriendo matices diferenciadores, dependiendo de las diferentes concepciones 0 modelos de universidad desarrollados. En estos últimos años Briscall (2000) propone la implementación en la enseñanza universitaria de la figura del docente tutor como un servicio fundamental de las universidades, donde una parte del profesorado o del tiempo que se destina a actividades docentes deberá asignarse a tareas de asesoramiento a los estudiantes. Ésta labor ha de ser: previa al ingreso en la Universidad, en la preparación y desarrollo de las habilidades educativas, en la planificación de los estudios, en situaciones que requieran apoyos especiales en casos de crisis o dificultades particulares, en el desenvolvimiento formativo, en la participación en la evolución y en la orientación profesional. Otros informes internacionales como el Delors (2003) y Attalli (1998) ofrecen una serie de recomendaciones sobre la necesidad de privilegiar en todos los casos la relación entre docente y alumno. También coinciden en señalar la necesidad de propiciar un mejor seguimiento y atención del alumnado universitario tanto al inicio, como durante los estudios y al finalizar estos.

La Ley Orgánica de Universidades señala que el alumnado tendrá derecho a asesoramiento y asistencia por parte de los tutores. Además, el Sistema de Transferencia de Crédito Europeo (ECTS) se centra en el aprendizaje del alumnado, como parte del proceso de enseñanza- 
aprendizaje al que hace referencia el Espacio Europeo de Educación Superior (Caballero, 2007). Por tanto, resalta la labor del tutor donde tendrá que incluir, además de las horas de docencia, las dedicadas a organizar, orientar y supervisar el trabajo del alumnado en el horario de tutoría. Se busca la progresión hacia una formación integral del alumnado que incluya los aspectos necesarios que, desde el inicio, le proporcione un punto de vista referencial útil en la toma de todas aquellas decisiones orientadas a desarrollar al máximo sus capacidades, destrezas, habilidades, así como a facilitar su integración y mantenimiento en la vida laboral. Esto va a requerir un esfuerzo añadido por parte del profesorado que, como señala Pagani (2002), con el nuevo sistema europeo "no sólo se deberán tener en cuenta las horas de docencia presenciales y tutorías, ya que los profesores, cuando se adopten los nuevos métodos docentes, tendrán que invertir un mayor tiempo en la preparación de sus asignatura y en la atención personalizada a los estudiantes".

Desde el punto de vista legal está claramente establecido el número de horas que el profesorado ha de dedicar a la labor tutorial. No obstante, a diferencia de lo que ocurre en otros niveles educativos, quedan sin especificar las funciones que ha de realizar durante ese tiempo el profesorado universitario. Debido probablemente, entre otros factores, a esta carencia normativa, algunos autores se han visto motivados a plantear sus aportaciones. A modo de ejemplo, Lázaro (1997) sitúa las funciones de un tutor universitario en dos grandes ámbitos educativos: intelectual y afectivo-madurativo. Homar (2002) indica que las tres funciones primordiales en el tutor son: informativa, seguimiento académico e intervención formativa y orientación. Alañon (2003) especifica que estas funciones serían: enseñar a estudiar (métodos de trabajo); motivar, ayudar y animar; orientar en problemas personales; orientar específicamente sobre la materia, y realizar tareas complementarias de formación sobre áreas de interés educacional general.

Otros autores se han centrado en explicitar bien las condiciones, cualidades, estilos, actitudes, o competencias que ha de poseer un tutor. Entre los primeros Zabalza (2003) destaca las siguientes condiciones personales: accesibilidad, locuacidad, credibilidad, paciencia, y condiciones materiales: espacio adecuado y número reducido de alumnos. La propuesta sobre cualidades personales que ha de reunir la figura del tutor es realizada por García y otros (2005) que proponen: personalidad con capacidad de influir positivamente en los demás, sensibilidad para captar y entender los problemas juveniles, y capacidad de entablar relaciones afectuosas y cordiales con los demás. Los autores añaden a estas cualidades personales, que aún 
siendo necesarias no son suficientes, otras cualidades de conocimiento como: saberes científicos, conocimientos teóricos sobre educación y ciencias afines; conocimientos teóricos y prácticos sobre relaciones interpersonales, dirección y animación de grupos, y conocimientos de técnicas de diagnóstico e intervención educativa. Entre los estudios sobre propuestas de diferentes estilos o actitudes de los tutores encontramos los de Brunet y Negro (1989) y Lázaro (1997) que señalan los estilos autoritario, democrático y que deja hacer, y los burocráticofuncionario, académico, docente y asesor personal respectivamente. Por último, Rodríguez (2004) considera que para llevar a la práctica sus roles y funciones, el profesor tutor ha de mostrar una actitud docente, colaborativa, participativa, comprensiva, comprometida, crítica y de ayuda personal. Por lo que respecta a lo que se consideran competencias imprescindibles en la función del tutor, García (2006) plantea: conocimientos sobre comunicación, habilidades o destrezas metodológicas para tratar con distintos colectivos y situaciones, actitudes como las de reconocer las habilidades y limitaciones propias en el proceso de ayuda, y actitud abierta al cambio y a la empatía.

Para la puesta en práctica de una acción tutorial que cubra la variedad de funciones propuestas tanto en la literatura como en la práctica diaria (De Miguel, Jiménez, Rodríguez y Macías, 2013), parece lógico que se haya ido implementando mediante diferentes tipos de tutorías, que han ido evolucionando para adaptarse a los fines de la institución universitaria. Rodríguez (2004) propone una síntesis de diferentes tipos de tutorías clasificándolas en función de una serie de criterios: servicios de orientación (modelo tutorial puro y mixto), contenido de las tutorías (de materia, de prácticas, de proyecto, de asesoramiento personal), figura del tutor (profesor-tutor y de iguales), tiempo (de curso, de carrera o de itinerario académico) y destinatarios (individual y grupal). Además, el mismo autor presenta una serie de características que definen a la tutoría universitaria: permite la integración activa del estudiante en el ámbito universitario; impulsa y facilita el desarrollo integral del alumnado en sus diferentes ámbitos: intelectual, afectivo, personal y social; contribuye a personalizar la educación universitaria, al realizar un seguimiento académico individualizado del alumnado en el itinerario de su formación; canaliza y dinamiza las relaciones del alumnado con diferentes segmentos de atención al estudiante: administrativo, docente, organizativo y de servicios, y la atención al estudiante constituye un elemento clave de calidad.

En palabras de Rodríguez (2004) "el tutor universitario es aquel profesor que ha de tener una motivación y preparación para la docencia así como un interés por el desarrollo del alumnado como 
persona, como estudiante y como futuro profesional". Y concreta en tres los principales roles del profesor-tutor universitario: profesor tutor de materia (facilita al alumnado información académica y hace un seguimiento y supervisión de sus procesos de aprendizaje), tutor de itinerario académico (tutor con tareas de información y orientación sobre aspectos de tipo profesional y del mundo laboral) y tutor de asesoramiento personal (tutor referente que presta atención individualizada a algunos alumnos que solicitan ayuda y mediación en aspectos relacionados con su desarrollo personal, educativo y profesional. Esta figura puede coincidir o no con el tutor de itinerario curricular).

Rose (2003) trabaja sobre una escala de mentor "ideal" en función de las expectativas del alumnado estudiante graduado. Consideramos que en nuestro contexto son escasos los estudios encontrados que analizan el punto de vista del alumnado ante la figura del tutor (Becker y Yager, 2013). Procede referir los realizados por Lázaro (1997, 2003). En el primero, el autor se centra en conocer las expectativas que tenían los estudiantes universitarios ante la figura del tutor. Destaca, entre otros resultados, la importancia que se le concede a la afectividad como condición indispensable para el ejercicio de la tutoría, la capacidad de comunicación empática y la competencia en el establecimiento de una relación interpersonal. En el segundo de los estudios, el autor establece las cinco cualidades que el alumnado considera que debe tener un tutor: Afectividad o capacidad de empatía, de mantener una relación en un clima de acogida, aunque manteniéndose en un punto intermedio, sin percepción de rechazo y sin manifestar un entusiasmo excesivo, individualización o conocer a sus estudiantes personalmente, ecuanimidad en el trato, autoridad serena, y respeto a todos los estudiantes.

La acción tutorial se ve inmersa en un profundo proceso de cambio. En este sentido, se ha considerado relevante conocer la opinión del alumnado, como parte implicada en el proceso, sobre cuáles son las competencias del profesorado tutor del siglo XXI, ante los cambios propuestos desde el marco de la Convergencia Europea.

El objetivo del estudio es conocer la opinión de alumnado universitario, de diferentes cursos académicos, en relación con las competencias que ha de desarrollar un tutor para ser considerado excelente. 
Competencias del docente universitario como guía en el espacio europeo de educación superior

\section{MÉTODO}

\subsection{Participantes}

Los 56 participantes cursaban asignaturas impartidas por profesorado adscrito al Departamento de Psicología Social de la Facultad de Psicología de la Universidad de Sevilla durante el curso académico 2010-2011.

Como la calidad de los resultados alcanzados con una metodología Delphi depende de la elección de los expertos y de su voluntad de participación, del compromiso a participar en todas las rondas que se establezcan (Cabero, 2014), se seleccionaron estudiantes que habían asistido a un mínimo de dos tutorías en su trayectoria universitaria, tenían conocimiento y experiencia del tema tutorial y, sobre todo querían participar.

Participaron un total de 56 alumnos, de los cuales el $77 \%$ eran mujeres y el $23 \%$ eran hombres, con edades comprendidas entre 19 y 23 años (Véase tabla 1).

\begin{tabular}{|c|c|c|c|c|c|}
\hline \multicolumn{6}{|c|}{ Grupos Delpfi } \\
\hline Curso & $1^{\circ}$ & $2^{\circ}$ & $3^{\circ}$ & $4^{\circ}$ & $5^{\circ}$ \\
\hline Asignatura & $\begin{array}{l}\text { Psicología } \\
\text { Social }\end{array}$ & $\begin{array}{l}\text { Psicología } \\
\text { Social de la } \\
\text { Comunicac. }\end{array}$ & $\begin{array}{c}\text { Psicología } \\
\text { Social Sistema } \\
\text { Educativo }\end{array}$ & $\begin{array}{l}\text { Psicología } \\
\text { de los } \\
\text { Grupos }\end{array}$ & $\begin{array}{c}\text { Intervención } \\
\text { Grupal }\end{array}$ \\
\hline Participantes & 15 & 8 & 12 & 11 & 10 \\
\hline Género & $10(M)$ & $8(M)$ & $10(M)$ & $8(M)$ & $7(M)$ \\
\hline
\end{tabular}

Tabla 1. Resumen de las características de los participantes o expertos.

\subsection{Diseño}

El diseño del estudio es descriptivo, en el cual los resultados son obtenidos utilizando la técnica Delphi que consta de distintas circulaciones.

\subsection{Instrumentos}

Para obtener los datos se emplearon varios instrumentos. En la primera ronda se elaboró una pregunta abierta que pretendía recabar la opinión del alumnado sobre qué competencias tendría que desarrollar un tutor para ser considerado excelente en el EEES. En una segunda ronda se elaboró un primer cuestionario, en función de las respuestas obtenidas en la primera ronda, donde los participantes 
debían valorar la importancia de las competencias que consideraban debería poseer un tutor para ser considerado excelente. Por último, se elaboró un segundo cuestionario donde el alumnado tenía que seleccionar y ordenar, de más a menos importantes, tres competencias contenidas en el primer cuestionario.

\subsection{Procedimiento}

Después de solicitar en el aula al alumnado la participación en el estudio, se estableció un primer contacto vía correo electrónico con los 83 alumnos que decidieron participar, donde se explicó de forma individual el procedimiento a seguir.

En la primera ronda se elaboró una pregunta abierta breve a la que el alumnado tenía que emitir tantas respuestas como consideraran necesarias. La pregunta era la siguiente: "Indica qué tendría que "saber" un tutor, qué tendría que "saber hacer" y cómo tendría que "saber ser y estar" para ser considerado excelente en el Espacio Europeo de Educación Superior".

Durante la segunda ronda Delphi se codificaron, mediante un análisis de contenido, las respuestas emitidas por el alumnado, que conformarían los ítems de competencias de un primer cuestionario. Cada participante tendría que determinar, mediante una escala tipo Likert, el grado de importancia que le concedía a cada competencia ( 1 poco importante - 5 muy importante) sobre cómo debería ser un tutor excelente.

Tras recibir cumplimentados los cuestionarios se inició la tercera ronda Delphi. En esta ronda se calcularon las medias y las desviaciones tipo de cada una de las competencias que componen el cuestionario. Estos descriptivos se utilizaron para elaborar un segundo cuestionario y, además, proporcionaron al alumnado retroalimentación sobre las respuestas emitidas, lo que ofreció la oportunidad de reevaluar sus respuestas si lo consideraban necesario. En esta ronda los participantes seleccionaron y ordenaron jerárquicamente tres de las competencias que componían el segundo cuestionario.

Como cuarta y última ronda Delphi se analizaron los datos del segundo cuestionario y se ofreció al alumnado un resumen del análisis de los resultados obtenidos. 
Competencias del docente universitario como guía en el espacio europeo de

\section{RESULTADOS}

De los cinco grupos Delphi que participaron en el estudio se obtuvieron un total de 92 competencias de la figura del tutor. De ellas 14 (15.2\%) hacían referencia a lo que tendría que "saber" (conocimientos), 37 (40.2\%) a lo que tendría que "saber hacer" (procedimientos) y 41 (44.6\%) a como debería "saber ser y estar" (actitudes personales y sociales). Se han destacado en "negrita" aquellas que fueron emitidas en más de dos ocasiones por el alumnado. Estas 53 competencias son las que se utilizarón para la elaboración del primer cuestionario Delphi. Las mismas corresponden a 6 (11.3\%) competencias de "saber", 24 (45.3\%) de "saber hacer" y 23 (43.4\%) de "saber ser y estar" (Véase tabla 2).

\begin{tabular}{|c|c|c|}
\hline \multicolumn{3}{|c|}{$\begin{array}{c}\text { ¿Qué tendría que "saber", "saber hacer" y "saber ser y estar" un futor para ser } \\
\text { considerado excelente en el contexto del EEES"? }\end{array}$} \\
\hline Saber & Saber hacer & Saber ser y estar \\
\hline $\begin{array}{l}\text { Dificultades académicas } \\
\text { Dificultades profesionales } \\
\text { Estrategias de aprendizaje } \\
\text { Estrategias de autoaprendizaje } \\
\text { Información de recursos } \\
\text { Materia que imparte } \\
\text { Orientación en el estudio } \\
\text { Problemas y planteamientos } \\
\text { de actualidad } \\
\text { Realidad laboral } \\
\text { Rendimiento académico } \\
\text { Técnicas de estudios } \\
\text { Tipos de tutorías } \\
\text { Características de la Titulación } \\
\text { Características de la } \\
\text { Universidad }\end{array}$ & $\begin{array}{l}\text { Aceptar críticas (trabajo que } \\
\text { desarrolla) } \\
\text { Aceptar diferentes puntos de } \\
\text { vista } \\
\text { Aplicar dinámicas de grupo } \\
\text { Apoyar desarrollo académico } \\
\text { Apoyar desarrollo personal } \\
\text { Apoyar desarrollo profesional } \\
\text { Confiar en el alumnado } \\
\text { (capacidades y } \\
\text { posibilidades) } \\
\text { Consensuar opiniones del } \\
\text { alumnado } \\
\text { Considerar diferencias } \\
\text { individuales } \\
\text { Desarrollar habilidades de } \\
\text { comunicación } \\
\text { Detectar intereses del } \\
\text { alumnado } \\
\text { Escuchar activamente } \\
\text { Estimular el aprendizaje } \\
\text { Estimular la motivación } \\
\text { Facilitar opiniones personales } \\
\text { Fomentar discusiones } \\
\text { grupales } \\
\text { Fomentar el aprendizaje } \\
\text { cooperativo } \\
\text { Fomentar la actividad tutorial } \\
\text { Identificar necesidades e } \\
\text { intereses } \\
\text { Mantener distancia } \\
\text { psicológica (tuteo) } \\
\text { Mantener interacciones cara } \\
\text { a cara (no detrás de la mesa) } \\
\text { Orientar elección de materias } \\
\text { Orientar itinerario académico } \\
\text { Orientar itinerario profesional } \\
\text { Potenciar conocimiento } \\
\text { Pomadion }\end{array}$ & 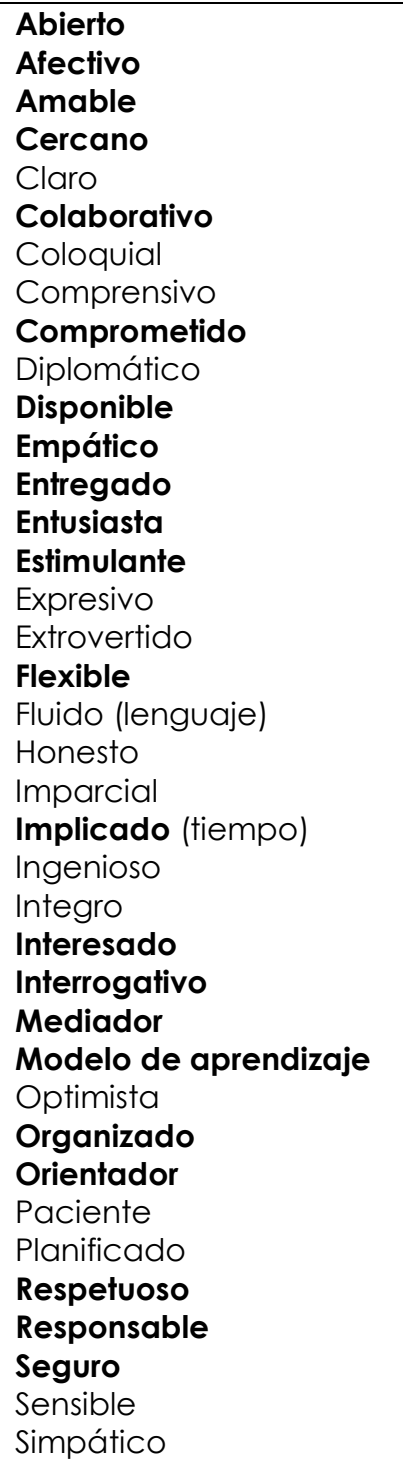 \\
\hline
\end{tabular}




\begin{tabular}{|c|c|c|}
\hline \multicolumn{3}{|c|}{$\begin{array}{c}\text { ¿Qué tendría que "saber", "saber hacer" y "saber ser y estar" un tutor para ser } \\
\text { considerado excelente en el contexto del EEES"? }\end{array}$} \\
\hline Saber & Saber hacer & Saber ser y estar \\
\hline & $\begin{array}{l}\text { personal } \\
\text { Realizar críticas constructivas } \\
\text { Realizar preguntas } \\
\text { Realizar recompensas } \\
\text { extrínsecas } \\
\text { Realizar trato individualizado } \\
\text { Regularizar contactos } \\
\text { tutoriales } \\
\text { Reorganizar las tareas } \\
\text { Resolver o gestionar conflictos } \\
\text { Respetar puntos de vistas } \\
\text { Retroalimentar } \\
\text { Revisar los trabajos con } \\
\text { criterios objetivos } \\
\text { Utilizar las tecnologías } \\
\text { (tutorías telemáticas) } \\
\text { Utilizar reforzadores positivos }\end{array}$ & $\begin{array}{l}\text { Sincero } \\
\text { Solidario } \\
\text { Tolerante }\end{array}$ \\
\hline
\end{tabular}

Tabla 2. Respuestas a la pregunta abierta.

Mediante el primer cuestionario se obtuvieron 23 competencias, que se destacan en "negrita", cuyas puntuaciones medias otorgadas son como mínimo 3.2, y que se encuentran entre los extremos 1 y 5 . De estas 23 competencias 3 (13\%) corresponden a "saber", 10 (43.5\%) a "saber hacer" y 10 (43.5\%) a "saber ser y estar" (Véase tabla 3). Procede señalar que dada la reducida cifra de competencias que hacen referencia a "saber", se mantuvieron, para la elaboración del segundo cuestionario Delphi, las seis competencias con la idea de que los tres grupos tuvieran una cifra similar.

\begin{tabular}{llcc}
\hline Primer & En tutor excelente debería.... & Media & D. T. \\
\hline \multirow{5}{*}{ Substionario } & Características de la Titulación & $\mathbf{4 . 4}$ & .76 \\
& Estrategias de aprendizaje & 3.1 & .66 \\
& Estrategias de autoaprendizaje & 3.0 & .75 \\
& Orientación en el estudio & 2.5 & .84 \\
& Realidad laboral & $\mathbf{3 . 7}$ & .53 \\
& Técnicas de estudios & 3.8 & .41 \\
\hline & Aplicar dinámicas de grupo & 3.5 & .68 \\
& Apoyar desarrollo académico & 2.6 & .40 \\
& Apoyar desarrollo profesional & 2.4 & .56 \\
& Confiar en el alumnado & 3.4 & .70 \\
& Desarrollar habilidades de comunicación & $\mathbf{4 . 4}$ & .34 \\
& Escuchar activamente & $\mathbf{4 . 0}$ & .53 \\
& Estimular el aprendizaje & 3.8 & .37 \\
& Estimular la motivación & 2.0 & .71 \\
& Fomentar discusiones grupales & 1.9 & .94 \\
& Fomentar el aprendizaje cooperativo & 2.1 & .58 \\
& Identificar necesidades e intereses & 3.3 & .46 \\
& Mantener interacciones cara a cara & 3.1 & .79 \\
& Orientar elección de materias & 3.0 & .83 \\
\hline
\end{tabular}


Competencias del docente universitario como guía en el espacio europeo de educación superior

\begin{tabular}{|c|c|c|c|}
\hline $\begin{array}{l}\text { Primer } \\
\text { Cuestionario }\end{array}$ & En futor excelente debería.... & Media & D. T. \\
\hline & Orientar itinerario académico & 3.8 & .41 \\
\hline & Orientar itinerario profesional & 3.7 & .51 \\
\hline & Realizar críticas constructivas & 2.6 & .56 \\
\hline & Realizar preguntas & 2.5 & .76 \\
\hline & Regularizar contactos tutoriales & 2.9 & .88 \\
\hline & Resolver o gestionar conflictos & 3.3 & .58 \\
\hline & Respetar puntos de vistas & 3.8 & .53 \\
\hline & Retroalimentar & 2.8 & .69 \\
\hline & Revisar los trabajos con criterios objetivos & 3.1 & .49 \\
\hline & Utilizar las nuevas tecnologías & 3.0 & .64 \\
\hline & Utilizar reforzadores positivos & 1.8 & .82 \\
\hline \multirow{23}{*}{$\begin{array}{l}\text { Saber ser y } \\
\text { estar }\end{array}$} & Abierto & 2.9 & .65 \\
\hline & Afectivo & 2.9 & .91 \\
\hline & Amable & 3.4 & .27 \\
\hline & Cercano & 3.7 & .46 \\
\hline & Colaborativo & 2.8 & .49 \\
\hline & Comprometido & 3.2 & .58 \\
\hline & Disponible & 3.0 & .53 \\
\hline & Empático & 4.5 & .44 \\
\hline & Entregado & 1.7 & .82 \\
\hline & Entusiasta & 2.0 & .93 \\
\hline & Estimulante & 3.3 & .50 \\
\hline & Flexible & 3.6 & .66 \\
\hline & Implicado (tiempo) & 3.9 & .49 \\
\hline & Interesado & 4.0 & .51 \\
\hline & Interrogativo & 2.6 & .68 \\
\hline & Mediador & 2.3 & .92 \\
\hline & Modelo de aprendizaje & 2.8 & .64 \\
\hline & Organizado & 3.1 & .68 \\
\hline & Orientador & 3.7 & .26 \\
\hline & Respetuoso & 4.3 & .47 \\
\hline & Responsable & 2.1 & .73 \\
\hline & Seguro & 2.5 & .58 \\
\hline & Sincero & 3.0 & .49 \\
\hline
\end{tabular}

Tabla 3. Respuestas al primer cuestionario.

A continuación se solicitó al alumnado que, en cada uno de los tres niveles competenciales, seleccionaran y priorizaran las tres competencias de mayor relevancia en el desempeño de la función tutorial. Las respuestas se agruparon considerando el curso que estaban cursando. Consideraron que principalmente, el tutor tiene que "saber" o tener conocimientos sobre las características de la titulación (en 4 cursos), sobre técnicas de estudio (en 3 cursos) y conocer la realidad laboral (en 3 cursos). En relación con lo que el tutor tiene que "saber hacer" destacaron que sea capaz de desarrollar habilidades de comunicación (en 3 cursos), de escuchar de manera activa (en 2 cursos) y de orientar tanto en el itinerario académico (en 2 cursos) como profesional (en 2 cursos). En lo que respecta a las competencias de 
"saber ser y estar" señalaron que el tutor tiene que ser empático (en 3 cursos), comprometido (en 3 cursos) y respetuoso con el alumnado (en 2 cursos).

La selección de competencias varía en función del curso en el que se encuentra el alumnado. Procede resaltar que, en el nivel competencial "saber" la competencia "Técnicas de estudio" presente en los dos primeros cursos deja paso a la competencia "Realidad laboral" en los dos últimos, apareciendo de manera simultánea en el tercero. En el nivel competencial "saber hacer" ocurre algo similar en cuanto a la competencia "Orientar itinerario académico" en primer y segundo curso y "Orientar itinerario profesional" en cuarto y quinto curso. Con respecto al nivel competencial "saber ser y estar" se plantea una demanda de la competencia del tutor "Respetuoso" en los dos primeros cursos frente a una demanda de la competencia del tutor "Empático" en los tres últimos cursos. Estas selecciones vienen graduadas por las diferentes prioridades asignadas por el alumnado (Véase tabla 4).

\begin{tabular}{|c|c|c|c|}
\hline \multirow{2}{*}{$\begin{array}{l}\text { Segundo } \\
\text { Cuestionario }\end{array}$} & \multicolumn{3}{|c|}{ Las principales competencias de un tutor excelente serían .... } \\
\hline & Saber & Saber hacer & Saber ser y estar \\
\hline $1^{\circ}$ Curso & $\begin{array}{l}\text { 1. Técnicas de } \\
\text { estudios } \\
\text { 2. Características } \\
\text { de la Titulación } \\
\text { 3. Orientación en el } \\
\text { estudio }\end{array}$ & 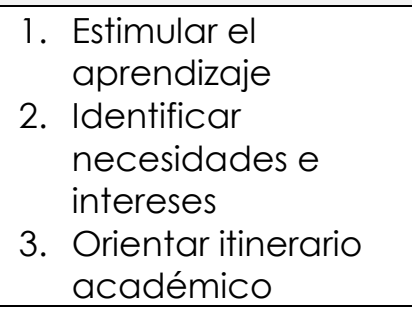 & $\begin{array}{ll}\text { 1. } & \text { Amable } \\
\text { 2. Estimulante } \\
\text { 3. }\end{array}$ \\
\hline $2^{\circ}$ Curso & $\begin{array}{l}\text { 1. Técnicas de } \\
\text { estudios } \\
\text { 2. Características } \\
\text { de la Titulación } \\
\text { 3. Estrategias de } \\
\text { autoaprendizaje }\end{array}$ & $\begin{array}{l}\text { 1. Confiar en el } \\
\text { alumnado } \\
\text { (capacidades y } \\
\text { posibilidades) } \\
\text { 2. Resolver o gestionar } \\
\text { conflictos } \\
\text { 3. Orientar itinerario } \\
\text { académico } \\
\end{array}$ & $\begin{array}{ll}\text { 1. } & \text { Cercano } \\
\text { 2. } & \text { Comprometido } \\
\text { 3. } & \text { Respetuoso }\end{array}$ \\
\hline $3^{\circ}$ Curso & $\begin{array}{l}\text { 1. Características } \\
\text { de la Titulación } \\
\text { 2. Técnicas de } \\
\text { estudios } \\
\text { 3. Realidad laboral }\end{array}$ & $\begin{array}{l}\text { 1. Desarrollar } \\
\text { habilidades de } \\
\text { comunicación } \\
\text { 2. Respetar puntos de } \\
\text { vista } \\
\text { 3. Escuchar } \\
\text { activamente } \\
\end{array}$ & $\begin{array}{l}\text { 1. Flexible } \\
\text { 2. Comprometido } \\
\text { 3. Empático }\end{array}$ \\
\hline $4^{\circ}$ Curso & $\begin{array}{l}\text { 1. Realidad laboral } \\
\text { 2. Estrategias de } \\
\text { aprendizaje } \\
\text { 3. Características } \\
\text { de la Titulación }\end{array}$ & $\begin{array}{l}\text { 1. Aplicar dinámicas } \\
\text { de grupo } \\
\text { 2. Desarrollar } \\
\text { habilidades de } \\
\text { comunicación } \\
\text { 3. Orientar itinerario } \\
\text { profesional }\end{array}$ & $\begin{array}{ll}\text { 1. Empático } \\
\text { 2. Comprometido } \\
\text { 3. }\end{array}$ \\
\hline
\end{tabular}


Competencias del docente universitario como guía en el espacio europeo de educación superior

\begin{tabular}{|c|c|c|c|}
\hline \multirow{2}{*}{$\begin{array}{l}\text { Segundo } \\
\text { Cuestionario }\end{array}$} & \multicolumn{3}{|c|}{ Las principales competencias de un futor excelente serían .... } \\
\hline & Saber & Saber hacer & Saber ser y estar \\
\hline $5^{\circ}$ Curso & $\begin{array}{l}\text { 1. Realidad laboral } \\
\text { 2. Estrategias de } \\
\text { aprendizaje } \\
\text { 3. Estrategias de } \\
\text { autoaprendizaje }\end{array}$ & $\begin{array}{l}\text { 1. Orientar itinerario } \\
\text { profesional } \\
\text { 2. Escuchar } \\
\text { activamente } \\
\text { 3. Desarrollar } \\
\text { habilidades de } \\
\text { comunicación }\end{array}$ & $\begin{array}{l}\text { 1. Empático } \\
\text { 2. Implicado } \\
\text { (tiempo) } \\
\text { 3. Interesado }\end{array}$ \\
\hline
\end{tabular}

Tabla 4. Respuestas al segundo cuestionario por cursos.

\section{CONCLUSIONES}

En general, los resultados encontrados permiten confirmar que las competencias que plantea el alumnado varían en función del curso que realiza. Por ello sería conveniente considerar en la ejecución de la acción tutorial o bien en los Planes de Acción Tutorial (PAT) las diferentes demandas que formula el alumnado para, de esta forma, ajustarse más a ellas. Sin embargo, este estudio que se ha centrado en conocer las competencias del tutor de Psicología, debería ampliarse, en principio, a tutores de otras áreas de conocimiento y también, en la línea planteada por García y otros (2005), consideramos interesante realizar estudios que analicen la percepción que tiene de la figura del tutor universitario el propio profesorado, qué competencias considera que tiene que desarrollar y cuáles desarrolla, sobre todo atendiendo a la importancia que se otorga a la educación integral del alumnado ante el Crédito Europeo.

Entendemos junto con Álvarez y Lázaro (2002), que el profesorado tiene ante si nuevos retos en un contexto de Convergencia Europea: el afrontar la diversidad del alumnado universitario, el acompañarle en sus procesos de aprendizaje y facilitarle un desarrollo integral que le prepare para la vida. Esto sólo se puede desarrollar si el profesorado asume la función tutorial como una parte de la función docente e investigadora.

Existe, por tanto, un continuum entre docencia y, tutoría y orientación. El profesorado tiene una función docente e investigadora, pero además debe afrontar una función tutorial. Todo educador, por el sólo hecho de serlo, asume una función orientadora aun sin ser plenamente consciente de ello. Sin embargo, no se trata de convertir a los docentes en orientadores. Se trata, simplemente, de hacer más explícita y con un mayor protagonismo la función que cada profesor desarrolla de forma espontánea como guía y facilitador del proceso de 
aprendizaje de su alumnado, tanto a nivel individual como grupal (García y Troyano, 2009).

Siguiendo la propuesta de Jiménez y Álvarez (2003), el camino a seguir por la tutoría universitaria en los próximos años sería apostar por la formación del tutor, por el reconocimiento de la labor tutorial, y por potenciar el aspecto humano de la relación tutorial. La acción tutorial requiere por tanto de un tiempo y una implicación personal que no siempre es valorada. Aún existen contextos, afortunadamente en regresión, en que se considera como un demerito más que como un mérito. Asumir la figura de tutor es una responsabilidad que supone la adquisición y el ejercicio de una serie de competencias. La mayoría del personal docente no ha recibido, sin embargo, una formación específica para ello. Dicha formación resulta básica para poder proporcionar respuestas adecuadas a las demandas que el alumnado puede ir planteando en su relación con la universidad e incrementar los recursos que permitan optimizar el desarrollo integral del alumnado tal y como plantea el EEES.

Cada tutor responde a una serie de competencias cognitivas, procedimentales y actitudinales personales y sociales que le diferencian del resto, lo que complica el poder definir con exactitud un perfil único (Troyano, García y Marín, 2006). Lo que sí parece claro es que el tutor ha de desarrollar una serie de competencias para lograr cubrir las demandas del alumnado, todo un reto que obliga a un replanteamiento de la acción tutorial. Habrán de tenerse en cuenta, entre otros, aspectos como las funciones tutoriales a desarrollar, el contexto en que se realizan o la experiencia. Se deberán analizar las prácticas y costumbres aprendidas o adquiridas, modificar o eliminar las que lo requieran, así como incorporar aquellas de las que se carezca. Ya que según la investigación realizada por Martínez, Pérez y Martínez (2014) los estudiantes de grado solicitan ante todo información, orientación en el itinerario formativo y atención al alumnado con necesidades específicas de apoyo educativo.

De cara a futuros trabajos, sería interesante profundizar en lo que Lozano, Ballesta, Alcaraz y Cerezo (2013) refieren en su trabajo sobre los canales de comunicación entre familia y escuela, llegando a comprender y valorar el papel que tiene la familia en el uso de las TIC y su importancia dentro del ámbito escolar, ya que las familias pueden condicionar el uso de estos medios a favor de los alumnos. De esta manera, el profesorado-tutor de ahora y del futuro habrá de contener además competencias tecnológicas que le permitan dar respuesta a las necesidades que plantea el alumnado en relación con las TIC. Este hecho supondrá que es necesario trabajar de forma coordinada 
Competencias del docente universitario como guía en el espacio europeo de educación superior

familia-escuela, puesto que la actitud de ambos colectivos va a influir en el desarrollo personal y social de nuestros alumnos.

\section{REFERENCIAS BIBLIOGRÁFICAS}

ALAÑON, M. T. (2003). Planificación, desarrollo y evaluación del Plan de Acción Tutorial en la Universidad. En P. Álvarez y H. Jiménez (Comp.). Tutoría Universitaria. La Laguna: Servicio de Publicaciones Universidad de La Laguna.

ÁLVAREZ, V. Y LÁZARO, Á. (Coords.) (2002). Calidad de las universidades y orientación universitaria. Málaga. Aljibe.

ATTALLI, J. et al. (1998). Pour un modéle européen dénseignement supérieur. Rapport de la Comisión présidée par Jacques Attali. Ed. Stock.

BECKER, A. Y YAGER, J. (2013). How to Approach Mentorship. The Academic Medicine Handbook: A Guide to Achievement and Fulfillment for Academic Faculty. Stanford, USA: Springer.

BRISCALL, J. M. (Coord.) (2000). Informe Universidad 2000. Madrid: Conferencia de Rectores de las Universidades Españolas (CRUE).

BRUNET, J. J. Y NEGRO, J. L. (1989). Tutoría con adolescentes. Madrid: Ediciones San Pío $X$.

CABALLERO, M.A. (2007). The European Higher Education Area (Bolonia process) as a Context for Reflection about Teaching-Learning Processes at University Level: Some Proposals for a Significant Change. REOP, 18 (2), 167-177.

CABERO, J. (2014). Formación del profesorado universitario en TIC. Aplicación del método Delphi para la selección de los contenidos formativos. Educación XX1, 17 (1), 111-132.

DOI: http://dx.doi.org/10.5944/educxx1.17.1.10707

DELORS, J. (2003). La educación encierra un tesoro. Informe a la UNESCO de la Comisión Internacional sobre Educación para el siglo XXI.

DE MIGUEL, C. R., JIMÉNEZ, E. G., RODRíGUEZ, S. R., \& MACÍAS, A. V. (2013). Innovación en la orientación Universitaria: la mentoría como respuesta. Contextos Educativos. Revista de Educación, 6, $87-112$.

GARCíA, A. (2006) La tutoría y la relación profesor-alumno en la formación para la inserción laboral. Revista de Educación, 341, 197-211.

GARCÍA, A.J. Y TROYANO, Y. (2009). El Espacio Europeo de Educación Superior y la figura del profesor tutor en la universidad. RED_U. Revista de Docencia Universitaria, 3, 1-10.

GARCÍA, J. (2012). Sistema emocional, función educativa y educación informal. Revista Fuentes, 12, 13-44. 
GARCÍA, N.; ASENSIO, I.; CARBALLO, R.; GARCÍA, M. Y GUARDIA, S. (2005). La tutoría universitaria ante el proceso de armonización europea. Revista de Educación, 337, 189-210.

HOMAR, J. M. (2002). La experiencia de tutorías universitarias en la Facultad de Formación del Profesorado. Disponible en http://www.uhu.es/fexp/tutorias/documentación.

JIMÉNEZ, H. Y ÁLVAREZ, P.R. (2003). La tutoría universitaria en España: aproximación a una variopinta realidad. En: P. Álvarez y $\mathrm{H}$. Jiménez (Comp.). Tutoría Universitaria. La Laguna: Servicio de Publicaciones Universidad de La Laguna.

LÁZARO, A. (1997). La acción tutorial de la función docente universitaria. En: P. Apodaca y C. Lobato. Calidad en la Universidad: orientación y evaluación. Barcelona: Laertes.

LÁZARO, A. (2003). Competencias tutoriales en la universidad. En F. Michavilla y F. García (Eds.). La tutoría y los nuevos modos de aprendizaje en la Universidad. Madrid: CAM- Cátedra UNESCO.

LOZANO, J., BALLESTA, F.J., ALCARAZ, S. Y CEREZO, M.C. (2013). Las tecnologías de la información y la comunicación en la relación familia-escuela. Revista Fuentes, 13, 173-192.

MARTíNEZ, P.; PÉREZ, J. Y MARTíNEZ, M. (2014). Una (re)visión de la tutoría universitaria: la percepción de estudiantes y tutores de estudios de Grado. Revista de Docencia Universitaria, 12 (1), 269-305.

PAGANI, R. (2002). El crédito europeo y el sistema educativo español. http://www.crue.org. espaeuro/encuentros/credito.pdf.

RODRíGUEZ, M. (2004). Represente el papel de tutor. En: L. M. Villar (Coord.). Programa para la mejora de la docencia universitaria. Madrid: Pearson Educación.

RODRÍGUEZ, S. (Coord.) (2004): Manual de Tutoría universitaria. Recursos para la acción. Barcelona: Octaedro/ICE-UB.

ROSE, G. L. (2003). Enhancement of mentor selection using the ideal mentor scale. Research in Higher Education, 44, 4, 473- 494.

D.O.I.: http://dx.doi.org/10.1023/A:1024289000849

TROYANO, Y., GARCÍA, A.J. Y MARÍN, M. (2006). ¿ Cómo afronta el profesorado universitario la docencia en el contexto de la convergencia europea?: hacia un nuevo perfil docente. Revista de Enseñanza Universitaria, 28, 77-83.

ZABALZA, M. A. (2003). Competencias docentes del profesorado universitario. Calidad y desarrollo profesional. Madrid: Narcea. 
Competencias del docente universitario como guía en el espacio europeo de educación superior

\section{Sobre los autores:}

\section{Alfonso Javier García González \\ alfonsoj@us.es}

Yolanda Troyano Rodríguez

ytroyano@us.es

Luís Sérgio Vieira

Isvieira@ualg.pt

Los doctores García, Troyano y Vieira mantienen una estrecha colaboración docente investigadora desde el año 2011. Han creado convenios académicos que les ha permitido realizar investigaciones sobre la influencia de la comunicación interpersonal del alumnado universitario en la mejora de sus habilidades sociales, el despliegue de respuestas asertivas en contextos profesionalizantes y la resolución de conflictos de manera eficaz. También cabe destacar la labor realizada por los tres en el campo de la orientación y la acción tutorial universitaria, desarrollando instrumentos de evaluación, coevaluación y autoevaluación de los procesos de orientación acometidos de forma inherente en la enseñanza-aprendizaje universitaria.

\section{Para citar este artículo:}

García, A.J.; Troyano, Y. y Vieira, L.S. (2014). Competencias del docente universitario como guía en el espacio europeo de educación superior. Revista Fuentes, 15, Junio, pp. 145-160. [Fecha de consulta: $\mathrm{dd} / \mathrm{mm} /$ aaaa]. http://www.revistafuentes.es/

D.O.I.: http://dx.doi.org/10.12795/revistafuentes.2014.115.07 